\title{
A STUDY OF LEAKAGE-CORRECTED TWO-STEP METHOD BASED ON THE NODAL EQUIVALENCE THEORY FOR FAST REACTOR ANALYSIS
}

\author{
Seongdong Jang ${ }^{1}$ and Yonghee Kim ${ }^{1}$ \\ ${ }^{1}$ Korea Advanced Institute of Science and Technology (KAIST) \\ 291 Daehak-ro, Yuseong-gu, Daejeon 34141, Republic of Korea \\ seongdong@kaist.ac.kr, yongheekim@kaist.ac.kr
}

\begin{abstract}
The conventional two-step method based on the generalized equivalence theory (GET) cannot be directly applied to the fast reactor analysis since the assumption of the spaceenergy separability is not very valid due to a relatively long neutron mean free path. This study aims to develop a leakage-corrected two-step method for the fast reactor analysis with the aid of the albedo-corrected parameterized equivalence constants (APEC) method. The critical idea of the APEC method is to correct the homogenized group constants (HGCs) including discontinuity factors (DFs) during the nodal calculation through predetermined APEC functions. The APEC functions are functionalized in terms of the normalized leakage parameters such as a current-to-flux (CFR) ratio so that they can correct the cross-sections (XSs) and discontinuity factors by reflecting the in-situ neutron leakage information of the nodal analysis. The feasibility of the APEC-corrected two-step method was investigated by solving 5-group diffusion equations for a two-dimensional sodium-cooled fast reactor with a 6-triangle finite difference method. The 5-group HGCs for fuel assemblies were determined by using a continuous-energy Monte Carl code, and the conventional assembly discontinuity factors are also introduced for each hexagonal fuel assembly. First of all, it was demonstrated that the simple FDM scheme could reproduce the reference nodal quantities with the GET. And the APEC functions are formulated using the reference solutions to evaluate the feasibility of simple APEC functional for both XSs and DFs. Then, a smaller color-set problem was defined to determine practical APEC functions for the original benchmark, and various numerical evaluations are performed in terms of the k-eff value and nodal power distribution.
\end{abstract}

KEYWORDS: APEC, Two-step Method, In-situ Leakage Correction, Fast Reactor Analysis

\section{INTRODUCTION}

The generalized equivalence theory (GET) is of huge importance in the state of the art reactor physics methods. The main idea of the GET is that transport-based node-wise and group-wise reaction rates can be preserved in a lower-order diffusion operator with the flux-volume-weighted homogenized group constants (HGCs) by introducing discontinuity factors (DFs) of the surface fluxes [1]. Consequently, even the simple diffusion nodal analysis can reproduce the reference nodal reaction rates, if the reference transport or Monte Carlo (MC) solution is known in advance.

Due to the limitation of the GET that the reference solution must be known in advance, the two-step methodology based on a simplified equivalence theory (SET) has been established as one of the cornerstones of the modern thermal reactor analysis. The procedure of the conventional two-step method 
is as follows: 1) the flux-volume-weighted HGCs and assembly-wise DFs (ADFs) are determined by transport-based pin-wise few group flux distribution, which is the solution of the two-dimensional lattice calculation, and 2) three-dimensional diffusion nodal analysis is performed. The two-step reactor analysis method assumes that the neutron flux is separable in terms of space and energy. In other words, the spacedependency of flux is separable from its energy dependency. The success of the conventional two-step method in thermal reactors indicates that each fuel assembly should have a somewhat similar asymptotic neutron spectrum regardless of its position in the light-water thermal reactors. The nice space-energy separability of neutron flux is primarily ascribed to the relatively short mean free path (MFP) of neutrons in the thermal reactors. However, the conventional two-step method has an inherent limitation when the neighborhood effect is rather strong.

In the modern fast reactor analysis, unfortunately, the accuracy of the conventional two-step methodology cannot be guaranteed since the neutron flux is hardly space-energy separable due to relatively long neutron MFP in the hard-spectrum reactors. As a result, it is necessary to take into account the positiondependent neutron spectrum when determining the multi-group HGCs for fast reactor fuel assemblies or zones. Consequently, a bit more complicated methods were developed. A well-known three-step method for fast reactor analysis is depicted in Fig. 1. In the typical 3-step method, the following calculations are performed: 1) zero-dimensional fine-group or continuous energy cross-section (XS) to determine the effective multi-group XSs, 2) two-dimensional R-Z neutron transport calculation with the effective multigroup XSs to obtain zone-wise neutron spectrums, which are subsequently used to condense the multigroup XSs into smaller group or few-group XS data, and 3) three-dimensional few-group whole-core diffusion nodal analysis [2]. The zone-wise spectrums for the group-condensation are approximately obtained by considering a simplified R-Z geometry of the original hexagonal core.

It is well perceived that over 10-energy groups are necessary for acceptable accuracy in the fast reactor analysis mainly due to relatively broad neutron spectrum and partially because of the rather simple and ad-hoc physics methods. To the authors' knowledge, there was no successful application of the conventional two-step method based on the GET in the fast reactor design and analysis due to the nonseparable space-energy dependency of the neutron flux. Meanwhile, Ref. 10 applied a simple two-step approach without the GET to two- and three-dimensional fast reactor analyses and showed that reasonable accuracy might be achieved with 24-group HGCs, which were determined by Monte Carlo lattice analyses.

Recently, the albedo-corrected parameterized equivalence constants (APEC) method was developed to correct lattice-based HGCs of the thermal reactors, XSs (diffusion coefficients, reaction XSs, and scattering XSs) and DFs, by accounting for neighboring effect through the actual leakage [3, 4]. The main principle of the APEC method is to allow the in-situ correction of HGCs during iteration of diffusion nodal analysis through the APEC function predetermined by color-set calculations. The APEC functions consist of normalized leakage parameters such as the current-to-flux (CFR) ratio to reflect the actual leakage of the three-dimensional diffusion nodal analysis. It was proven that in-situ corrections of HGCs could substantially improve the nodal solution of the thermal reactor analysis even for partially MOXloaded PWRs. The APEC method also proved to be effective in the middle of the burnup calculations [5, $6,7]$.

The purpose of the present study was to expand the concept of the leakage-corrected two-step method to the fast reactor analysis. As a preliminary study, the applicability of the APEC-corrected two-step method is mainly discussed in this work by solving a two-dimensional simplified prototype gen-IV sodium-cooled fast reactor (sPGSFR) with the newly proposed two-step method. In this work, the two-dimensional whole-core and color-set calculations are conducted by the Serpent 2 Monte Carlo code with the ENDF/B-VII.1 data library [8]. Then, a 5-group nodal diffusion analysis is performed by using a 6triangle finite difference method (FDM) with in-situ APEC corrections for the XSs and DFs values in each hexagonal fuel assembly. 


\section{APEC-CORRECTED TWO-STEP METHODOLOGY FOR FAST REACTOR ANALYSIS}

\subsection{APEC-corrected Two-step Method}

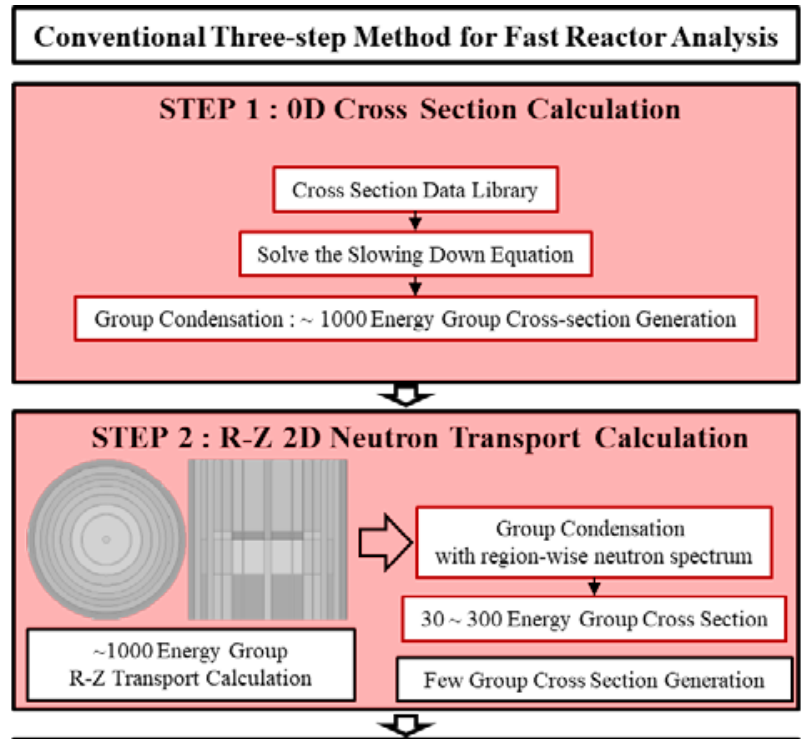

STEP 3 : 3D Whole Core Nodal Calculation (Diffusion)

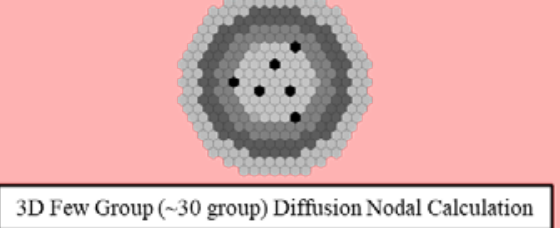

APEC-corrected Two-step Method for Fast Reactor Analysis

STEP 1 : Lattice / Color-set Calculation (Transport)

All Reflective BC

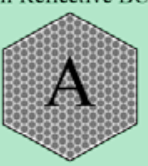

Single Assembly $\quad 1 / 12$ Core Color-se

Lattice Calculation Calculation

Data Library Files Generation for $5 \sim 10$ Group Constant and APEC Functions

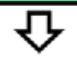

STEP 3 : 3D Whole Core Nodal Calculation (Diffusion)

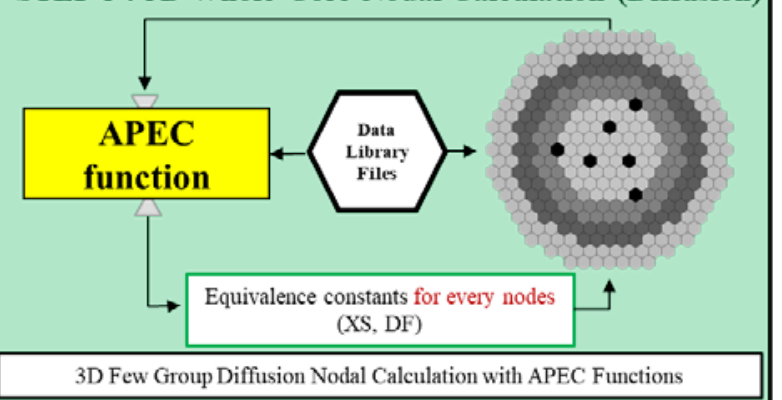

Figure 1. Comparison between Conventional Three-step and APEC-corrected Two-step Methods.

As shown in Fig. 1, the critical idea of the APEC-corrected two-step method is to determine the APEC functions using the results of the lattice and twelfth core color-set calculations, which are conducted with the Serpent 2 code in this work. The single lattice calculations provide the conventional homogenized flux-weighted group constants (FWCs) and ADFs for each fuel assembly. At the same time, the color-set analysis determines the reference position-dependent HGCs and DFs accounting for the non-zero interface neutron currents. Then, the HGCs differences $(\triangle X S$ and $\triangle D F)$ between the lattice and color-set models can be parameterized for the APEC functions in terms of the group-wise normalized neutron leakages, CFRs. Definitions of the color-set problems are dependent on the number of different fuel assemblies in the problem. In principle, the number of color-sets is to be minimized to optimize the computing costs. Given the lattice HGCs, $\triangle X S$, and $\triangle D F$, the APEC functions are determined by simple multiple linear regression procedures. Finally, the predetermined APEC functions can correct HGCs depending on the corresponding CFRs during the multi-dimensional nodal calculation to improve the nodal equivalence of the 2 -step fast reactor analysis.

The APEC HGCs functions consist of APEC XS and DF functions. For a given fuel assembly, a different APEC function is defined for each group and type of the HGCs: diffusion coefficients (D), removal XSs $\left(\Sigma_{r}\right)$, fission neutron production XSs $\left(v \Sigma_{f}\right)$ and scattering XS $\left(\Sigma_{s 0}\right)$, and DFs. The conventional reactor criticality calculation is done by the power method employing inner-outer nested iterations, and the HGCs are updated using the latest group-wise CFR information for each node and surface of the hexagonal fuel assemblies in each outer iteration. The APEC corrections can be similarly applied to the reflector HGCs without any problems. However, a fixed reflector HGCs are obtained for a spectral geometry without any 
leakage correction since the reflector design is supposed to be fixed for a given reactor. In the conventional fast reactor analysis, 10 30-group diffusion calculations are usually performed to achieve acceptable accuracy. It is expected that the number of energy groups can be reduced significantly with the aid of the APEC correction without compromising the accuracy. In this regard, a 5-group XS data is tested for a conventional sodium-cooled fast reactor analysis in this work.

\subsection{APEC XS function}

The XS can be defined in terms of the FWC XS and the $\triangle X S$, as shown in Eq. (1). The $\triangle X S$, which is called APEC XS function, can be functionalized in terms of the actual group-wise normalized leakage parameters, node-wise CFRs.

$$
\begin{gathered}
\Sigma_{x, g}=\sum_{x, g}^{F W C}+\Delta \Sigma_{x, g} \\
\Delta \Sigma_{x, g}^{A P E C}=\left\{\begin{array}{l}
a_{g, 1} \frac{\sum \bar{J}_{g}^{s}}{\sum \widehat{\phi}_{g}^{s}}+a_{g, 2} \frac{\sum \bar{J}_{g+1}^{s}}{\sum \widehat{\phi}_{g+1}^{s}}(1 \leq g \leq G-1) \\
a_{g, 1} \frac{\sum \bar{J}_{g}^{s}}{\sum \widehat{\phi}_{g}^{s}}+c_{g}
\end{array}\right.
\end{gathered}
$$

where,

$$
\begin{aligned}
& \Sigma_{x, g}: g^{\text {th }} \text { group, } x \text { type } X S, \\
& \Sigma_{x, g}^{F C}: g^{\text {th }} \text { group, } x \text { type } X S(F W C), \\
& \bar{J}_{g}^{s}: \text { surface average net current, } \\
& \hat{\phi}_{g}^{s}: \text { surface average flux. }
\end{aligned}
$$

The APEC XS functions are taking into account the fast reactor physics. The group-wise XSs might be affected by not only the within-group leakage through the surface due to the neighboring effect but also the down scattering in the medium. To reflect the impact of down scattering indirectly, a lower energy group CFR is additionally chosen as an independent variable for APEC XS functions. Regarding the last group, the APEC XS function is defined as a linear function of the within-group CFR only since the effect might be minor due to the hard spectrum of the fast reactor.

\subsection{APEC DF function}

Similarly to the definition of XS, the DF can be defined in terms of ADF and $\triangle D F$ as shown in Eq. (4). To reflect the characteristic of the DFs, which are determined differently depending on the surface, surfacewise leakage parameter, surface-wise CFR, is added as shown in Eq. (5).

$$
\begin{gathered}
\mathrm{DF}_{g}=A D F_{g}+\Delta D F_{g} \\
\Delta D F_{g}=a_{g, 1} \frac{\bar{J}_{g}^{s}}{\hat{\phi}_{g}^{s}}+a_{g, 2} \frac{\sum \bar{J}_{g}^{s}}{\sum \widehat{\phi}_{g}^{s}}+c_{g}
\end{gathered}
$$

It should be noted that DFs are the methodology-dependent parameters, and they should be evaluated depending on the nodal method in place. In this study, a 6-triangle FDM was used as a nodal method, so there are only six DFs at the surface of a hexagonal node. It is worthwhile to note that two kinds of CFRs are utilized for better DF modeling in Eq. (5). It is important to recognize that DFs are unity at the inner interface between triangular nodes in a hexagonal node. 


\section{NUMERICAL RESULTS}

\subsection{Nodal equivalence theory in fast reactor}

The two-dimensional sPGSFR benchmark problem was selected to evaluate the applicability of the APEC-corrected two-step method for the fast reactor analysis. It consists of 7-ring FAs and 2-ring reflector, as shown in Fig. 2. Each FA has 9-ring fuel pins that have 19.04 w/o enrichment [9]. The sPGSFR consists of a single FA type in the active core region. To apply APEC functions effectively, the two-dimensional sPGSFR core was divided into three HGCs groups, which are interior FA (IFA), peripheral FA (PFA), and the reflector region. It is noted that the reference XSs and DFs are used for the reflector region to investigate the effects of the APEC method. Since the reflector design is usually fixed in typical fast reactors, very reliable HGCs for the reflector region is feasible by solving the whole core problem only once.
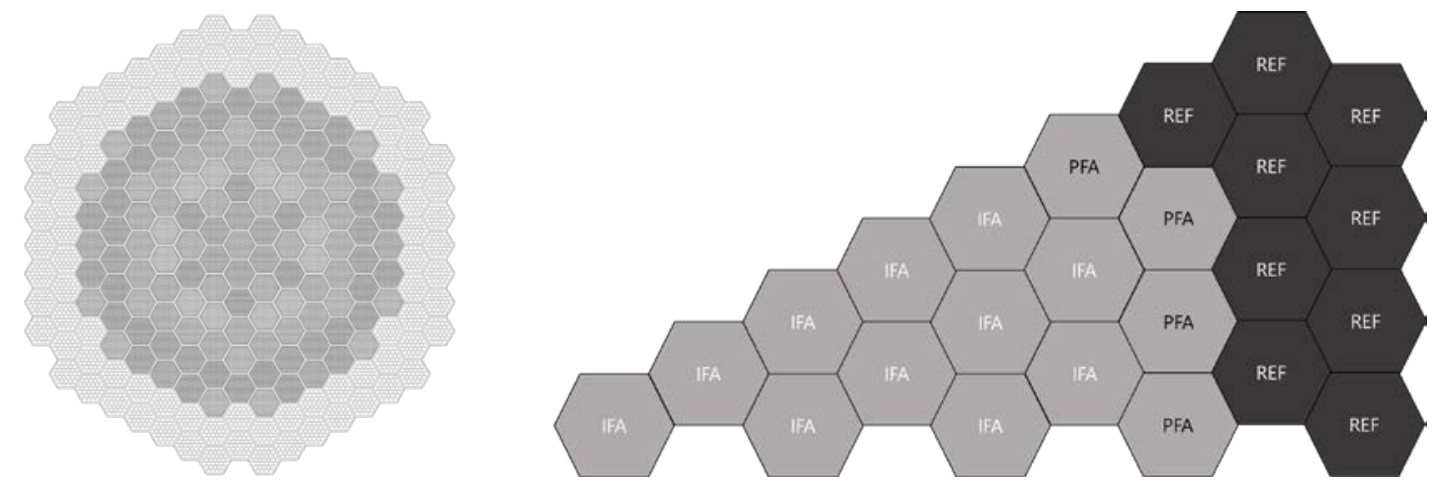

Figure 2. Two-dimensional Simplified Prototype Gen-IV Sodium-cooled Fast Reactor (sPGSFR) Benchmark Problem. (IFA: interior FA, PFA: peripheral FA, REF: reflector)

The reference whole core calculation was conducted by the Serpent 2 code with the Monte Carlo conditions of $50 \mathrm{M}$ histories, 300 inactive and 1000 active cycles. In this study, a 5-energy group structure was selected for the evaluation of in-situ leakage correction by the APEC method. The energy group boundary are $1.00 \mathrm{E}-10,4.54 \mathrm{E}-04,5.58 \mathrm{E}-03,1.12 \mathrm{E}-01,1.53 \mathrm{E}+00$, and $2.10 \mathrm{E}+01 \mathrm{MeV}$.

Table I. Results of nodal equivalence theory for the sPGSFR problem.

\begin{tabular}{|c|c|c|c|c|c|}
\hline $\mathrm{XS}$ & DF & $\mathrm{k}_{\text {eff }}$ & $\Delta \rho(\mathrm{pcm})$ & $* \operatorname{RMS}(\%)$ & $* * \operatorname{Max}(\%)$ \\
\hline \multicolumn{2}{|c|}{ Serpent 2} & 1.255598 & & & \\
\hline FWC & No & 1.262836 & 456.51 & 0.78 & 1.43 \\
\hline FWC & ADF & 1.255330 & -16.94 & 1.79 & -4.55 \\
\hline FWC & Ref. & 1.252731 & -182.23 & 0.35 & -1.36 \\
\hline Ref. & No & 1.265848 & 644.93 & 1.13 & 3.02 \\
\hline Ref. & ADF & 1.258266 & 168.90 & 1.44 & -3.04 \\
\hline Ref. & Ref. & 1.255606 & 0.53 & 0.04 & 0.19 \\
\hline
\end{tabular}

Using the reference solution, the reference DFs were calculated by solving augmented fixed-source problems by an in-house FDM code, which consists of 6-triangle mesh per a hexagonal node. Table I summarizes nodal performances depending on the XSs and DFs values. One notes that the reference solution can be reproduced by the GET, if reference HGCs are known. It should be noted that the conventional two-step with FWC XSs and ADFs results in relatively high errors in the power profile, while the k-eff prediction is rather accurate. The accurate k-eff prediction with the FWC and ADF is 
considered to be rather coincident due to error cancellation. On the other hand, a substantial error occurred when the DFs are ignored. Table I indicates that the DF is critical in achieving nodal equivalence in the fast reactor analysis, and both XS and DF should be accurate for a high fidelity solution. In addition, results in Table I demonstrate the feasibility of the simple two-step approaches.

To verify the basic feasibility of the APEC XS and DF functions, the reference solution was used to determine a set of reference APEC functions, and the resulting APEC functions were applied to the 5group diffusion-based FDM analysis for the benchmark and the results are summarized in Table II.

Table II. Application of reference APEC XS and DF functions.

\begin{tabular}{|c|c|c|c|c|c|}
\hline XS & DF & $\mathrm{k}_{\text {eff }}$ & $\Delta \rho(\mathrm{pcm})$ & $* \operatorname{RMS}(\%)$ & $* * \operatorname{Max}(\%)$ \\
\hline \multicolumn{2}{|c|}{ Serpent 2 } & 1.255598 & \multicolumn{3}{l|}{} \\
\hline FWC & Ref. APEC & 1.252794 & -178.22 & 0.39 & -1.66 \\
\hline Ref. APEC & ADF & 1.258167 & 162.68 & 1.65 & -5.11 \\
\hline Ref. & Ref. APEC & 1.255665 & 4.29 & 0.02 & -0.08 \\
\hline Ref. APEC & Ref. & 1.255613 & 0.97 & 0.39 & -1.69 \\
\hline Ref. APEC & Ref. APEC & 1.255675 & 4.93 & 0.44 & -1.90 \\
\hline
\end{tabular}
*oot Mean Square Error in Assembly Power (\%), ** Maximum Error in Assembly Power (\%)
†Reference XS and DF were used to reflector region

Table II shows that proposed APEC functional forms can correct the HGCs very substantially. Nevertheless, almost $2 \%$ maximum error occurs at the peripheral region when the reference APEC XS and DF are applied simultaneously since the accuracy of corrected HGCs of the PFA is not good enough. Both Tables I and II clearly attest to that the APEC DF correction is critically important for accurate 2step nodal solution. And one also observes that the APEC correction is very applicable to fast reactors when the APEC functions are appropriately determined in the form of Eqs. (1) to (5).

\subsection{APEC-corrected two-step method based on color-set calculation}

In the practical application of the APEC method, the reference solution is definitely unavailable, and the proper color-set problems are needed to determine the APEC functions. In this preliminary study, a smallsize core in Fig. 3 was chosen as the color-set model to produce a set of data for the APEC functions. Obviously, the smaller the color-set problem, the better in view of the computing cost. The actual computing cost for the color-set is rather small if a twelfth core problem is calculated.
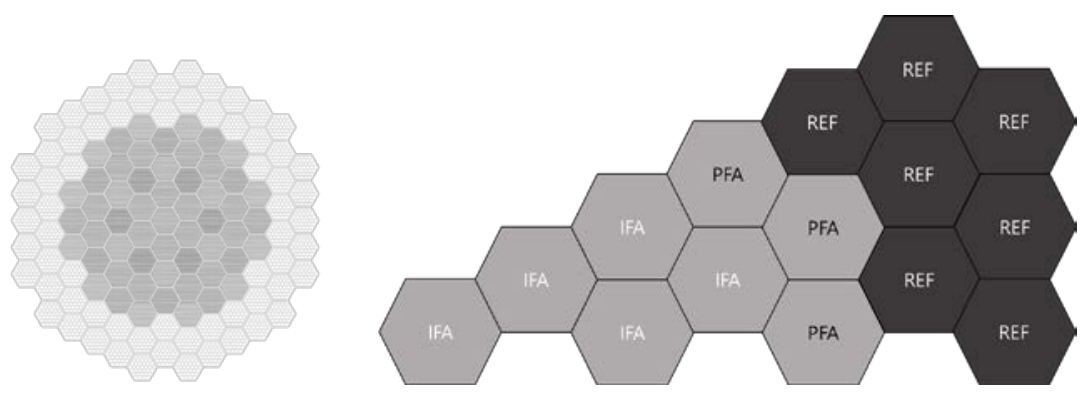

Figure 3. Two-dimensional Color-set Problem (IFA: interior FA, PFA: peripheral FA, REF: reflector)

In the sPGSFR benchmark, there is only one FA type and the above color-set can provide enough data to determine the APEC functions for two regions. If more FA types are considered, more color-sets or more complicated color-set configurations will be necessary to obtain reliable APEC functions for both XS and DF corrections. Results with the color-set-based APEC functions are presented in Table III and Fig. 4. 
Table III. Results of color-set-based APEC functions.

\begin{tabular}{|c|c|c|c|c|c|}
\hline $\mathrm{XS}$ & $\mathrm{DF}$ & $\mathrm{k}_{\text {eff }}$ & $\Delta \rho(\mathrm{pcm})$ & $* \mathrm{RMS}(\%)$ & $* * \operatorname{Max}(\%)$ \\
\hline \multicolumn{2}{|c|}{ Serpent 2} & 1.255598 & \multicolumn{3}{|c|}{} \\
\hline FWC & APEC & 1.252709 & -183.64 & 0.74 & -2.72 \\
\hline APEC & ADF & 1.258034 & 154.25 & 1.66 & -5.12 \\
\hline Ref. & APEC & 1.255584 & -0.87 & 0.38 & -1.10 \\
\hline APEC & Ref. & 1.255479 & -7.54 & 0.39 & -1.70 \\
\hline APEC & APEC & 1.255437 & -10.18 & 0.79 & -2.97 \\
\hline
\end{tabular}

* Root Mean Square Error in Assembly Power (\%) ** Maximum Error in Assembly Power (\%) $\dagger$ Reference $X S$ and $D F$ were used to reflector region.

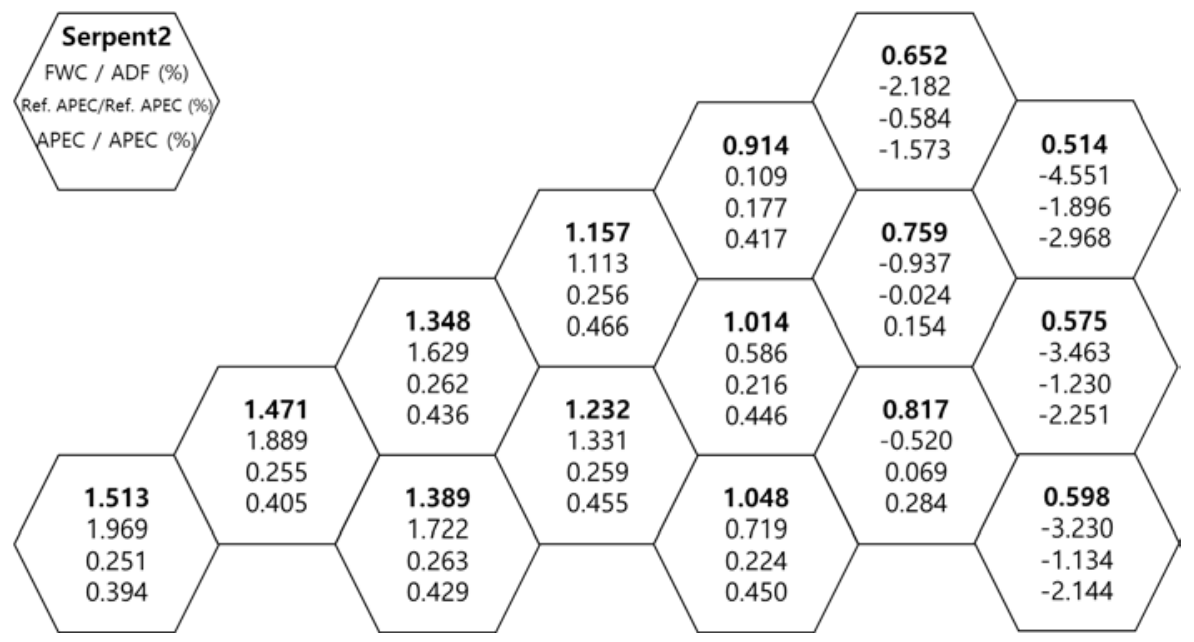

Figure 4. Radial Power Distribution of sPGSFR and Relative Power Error.

One can observe that the APEC functions generated by color-set can also well correct the HGCs of the sPGSFR, although maximum power error is about $3 \%$ at the peripheral region. It is important to note that the APEC-corrections result in clearly improved k-eff and interior power profiles. It can also be noted that APEC XS correction is more effective than the APEC DF correction for this benchmark.

Table IV. *RMS error of HGCs by SET and APEC correction.

\begin{tabular}{|c|c|c|c|c|c|c|c|c|}
\hline Energy & D & $v \Sigma_{f}$ & $\Sigma_{r}$ & $\sum_{s 0, E \rightarrow 2}$ & $\sum_{s 0, E \rightarrow 3}$ & $\Sigma_{s 0, E \rightarrow 5}$ & $\Sigma_{s 0, E \rightarrow 5}$ & DF \\
\hline \multicolumn{8}{|c|}{ HGCs by SET (FWC, ADF) } \\
\hline $1 \mathrm{~g} / 5 \mathrm{G}$ & $1.97 \mathrm{E}-01$ & $1.01 \mathrm{E}-02$ & $9.76 \mathrm{E}-03$ & $5.89 \mathrm{E}-03$ & $4.64 \mathrm{E}-04$ & $9.30 \mathrm{E}-06$ & $2.51 \mathrm{E}-06$ & $2.62 \mathrm{E}+00$ \\
\hline $2 \mathrm{~g} / 5 \mathrm{G}$ & $9.90 \mathrm{E}-01$ & $5.73 \mathrm{E}-04$ & $1.66 \mathrm{E}-02$ & - & $1.58 \mathrm{E}-02$ & $1.75 \mathrm{E}-05$ & $5.68 \mathrm{E}-07$ & $1.83 \mathrm{E}+00$ \\
\hline $3 \mathrm{~g} / 5 \mathrm{G}$ & $1.43 \mathrm{E}-01$ & $7.38 \mathrm{E}-03$ & $1.74 \mathrm{E}-02$ & - & - & $8.06 \mathrm{E}-03$ & $6.96 \mathrm{E}-07$ & $1.76 \mathrm{E}+00$ \\
\hline $4 \mathrm{~g} / 5 \mathrm{G}$ & $9.69 \mathrm{E}-01$ & $1.85 \mathrm{E}-01$ & $2.09 \mathrm{E}-01$ & - & - & - & $5.17 \mathrm{E}-02$ & $3.72 \mathrm{E}+00$ \\
\hline $5 \mathrm{~g} / 5 \mathrm{G}$ & $1.20 \mathrm{E}+00$ & $8.06 \mathrm{E}-01$ & $7.54 \mathrm{E}-01$ & - & - & - & - & $1.45 \mathrm{E}+01$ \\
\hline \multicolumn{8}{|c|}{ Converged HGCs by APEC method (APEC XS, APEC DF) } \\
\hline $1 \mathrm{~g} / 5 \mathrm{G}$ & $5.93 \mathrm{E}-02$ & $1.99 \mathrm{E}-03$ & $2.82 \mathrm{E}-03$ & $2.05 \mathrm{E}-03$ & $1.08 \mathrm{E}-04$ & $5.14 \mathrm{E}-06$ & $5.09 \mathrm{E}-06$ & $8.79 \mathrm{E}-01$ \\
\hline $2 \mathrm{~g} / 5 \mathrm{G}$ & $3.92 \mathrm{E}-02$ & $8.08 \mathrm{E}-05$ & $1.43 \mathrm{E}-03$ & - & $1.29 \mathrm{E}-03$ & $3.55 \mathrm{E}-06$ & $1.26 \mathrm{E}-07$ & $1.38 \mathrm{E}+00$ \\
\hline $3 \mathrm{~g} / 5 \mathrm{G}$ & $2.26 \mathrm{E}-02$ & $6.31 \mathrm{E}-04$ & $1.96 \mathrm{E}-03$ & - & - & $1.02 \mathrm{E}-03$ & $9.23 \mathrm{E}-07$ & $9.54 \mathrm{E}-01$ \\
\hline $4 \mathrm{~g} / 5 \mathrm{G}$ & $3.88 \mathrm{E}-02$ & $5.78 \mathrm{E}-03$ & $5.90 \mathrm{E}-03$ & - & - & - & $1.42 \mathrm{E}-03$ & $4.40 \mathrm{E}+00$ \\
\hline $5 \mathrm{~g} / 5 \mathrm{G}$ & $3.83 \mathrm{E}-01$ & $9.65 \mathrm{E}-02$ & $1.11 \mathrm{E}-01$ & - & - & - & - & $1.14 \mathrm{E}+01$ \\
\hline
\end{tabular}

* Root Mean Square Error of HGCs (\%) 
Table IV shows the RMS errors of the lattice HGCs and APEC-corrected ones at the end of the nodal calculation. It is obvious that all the group-wise XSs are corrected quite much by the APEC XS functions, while the APEC DF correction is rather limited. One also notes that the RMS DF errors for the $4^{\text {th }}$ and $5^{\text {th }}$ groups are rather high and hardly affected by the APEC corrections.

\section{CONCLUSIONS}

A preliminary study of an APEC-based in-situ leakage-correction method has been performed to develop an efficient and accurate two-step method for fast reactors. A 2-D sodium-cooled fast reactor was analyzed by a 6-triangle finite difference nodal method for 5-group diffusion equations corrected by the APEC functions. The numerical results show that the APEC-corrected two-step method based on the equivalence theory could clearly improve the nodal solution. The homogenized XSs for hexagonal fuel assemblies are very well corrected by the APEC functions predetermined using a simple color-set model. However, it was also found that the 5-group DFs were not well corrected for all groups for the simple FDM nodal method. It is expected that the two-step method may work quite well even with very few group models, e.g., 5-group for fast reactors if the leakage-correction can be done properly. As future work, it is necessary to optimize the APEC functional forms for the homogenized group constants for both fuel assemblies and reflector nodes in fast reactors.

\section{ACKNOWLEDGMENTS}

This work was supported by the National Research Foundation of Korea (NRF) Grant funded by the Korean Government (MSIP) (NRF-2016R1A5A1013919)

\section{REFERENCES}

1. K. S. SMITH, "Assembly Homogenization Techniques for Light Water Reactor Analysis," Prog. in Nucl. Energy, 17, 3, 303 (1986)

2. C. H. Lee and W. S. Yang, "MC2-3: Multigroup Cross Section Generation Code for Fast Reactor Analysis", Argonne National Laboratory report, ANL/NE-11-41 Rev.2, November 08, (2013)

3. W. Kim, W. Heo, Y. Kim, "Improvement of nodal accuracy by using albedo-corrected parameterized equivalence constant," Nucl. Sci. Eng. 188 (2017) 207-245

4. W. Kim, K. Lee, Y. Kim, "Functionalization of the discontinuity factor in the albedo-corrected parameterized equivalence constant (APEC) method," Nucl. Sci. Eng. 192 (2018) 1-20

5. K. Lee, W. Kim, Y. Kim, "Improved nodal equivalence with leakage-corrected cross sections and discontinuity factors for PWR depletion analysis," Nuclear Engineering and Technology, (2019)

6. S. Jang, and Y. Kim "Improved Discontinuity Factor Modeling for the in-situ APEC Leakage Correction in Nodal Analysis," Transactions of ANS Winter, November 17-21, Washington, D.C., (2019)

7. S. Jang, K. Lee and Y. Kim "APEC-corrected NEM Analysis of the VERA Core," proceedings of the Reactor Physics Asia 2019 Conference, December 2-3, Osaka, Japan, (2019)

8. J. LEPPÄNEN, "Serpent-a continuous-energy Monte Carlo reactor physics burnup calculation code," VTT Technical Research Centre of Finland, 4 (2013).

9. Chihyung Kim, Donny Hartanto, and Yonghee Kim, "Uranium Enrichment Reduction in the Prototype Gen-IV Sodium-Cooled Fast Reactor (PGSFR) with PBO Reflector," Nuclear Engineering and Technology, Vol. 48, p. 351-359, 2016. (6)

10. Tung Dong Cao Nguyen, Hyunsuk Lee, Xianan Du, Vutheam Dos, Tuan Quoc Tran and Deokjung Lee "MCS Multi-group Cross Sections Generation for Fast Reactor Analysis," proceedings of the Reactor Physics Asia 2019 Conference, December 2-3, Osaka, Japan, (2019) 